\section{The Ideal Romantic Partner Morality}

\author{
Ryo Oda*, Kanako Hayashi \\ Nagoya Institute of Technology, Nagoya 466-8555, Japan \\ "Author for correspondence (oda.ryo@nitech.ac.jp)
}

Assortative mating must be important for maintaining morality in a population, as moral principles are shared by most people in a group. Breeding by a pair with similar morals results in genetic and cultural transmission of these morals to the next generation, which maintains the moral norms of the group. In this study, we investigated absolute and relative mate preferences in relation to particular moral foundations, as represented by five general moral values. In both sexes, correlations between ratings for self and an ideal romantic partner on these factors were rather high $(.67 \leq r \leq .84)$. Differences between self-ratings and ratings for the ideal romantic partner did not deviate significantly from zero for any of these factors.

\section{Keywords}

assortative mating, moral, mate preference, ideal romantic partner, moral foundations

\section{Introduction}

Moral principles should be shared by most people in a group. Although there are some universal aspects, moral judgments differ among groups (e.g., Haidt et al., 1993). One reason for this could be that people with similar morals tend to gather together and their values are transmitted from generation to generation. Indeed, people tend to prefer contact with those who are similar to them in cultural, behavioral, or genetic aspects over contact with dissimilar people (McElreath et al., 2003; McPherson et al., 2001). Such homophily could contribute to the foundation and maintenance of morality. An important factor is assortative mating, which can be defined as the nonrandom coupling of individuals based on their resemblance to each other with regard to one or more characteristics (Buss, 1984). Because men and women have to cooperate to produce and raise helpless and time-consuming offspring, agreement among partners not only in terms of fidelity but also in many other aspects of morality is needed for successful breeding and increased fitness in men and women. Moreover, breeding by a pair with similar morals results in genetic and cultural transmission of these morals to the next generation, which would maintain the moral norms of a group. A possible mechanism of this maintenance is niche construction (Laland et al., 2001), which occurs when organisms alter the local environment to which they have adapted. Humans, as social animals, tend to construct social niches by actively choosing to form relationships that are congenial and low in conflict so as to construct a rewarding environment that fits their needs (Yamagishi \& Hashimoto, 2016). Morality is a principle shared by most people in a group; it can be regarded as a social environment to which members of the group are motivated to adapt. If people who share the same moral sense gather by assortative mating, a specific moral sense would tend to be reinforced and maintained in the group through construction of a social niche.

Many moral virtues may have evolved in both sexes to advertise good genetic quality, parenting ability, and/ or partner traits (Miller, 2007). Previous studies suggest that people desire kind, cooperative, and moral mates. In a large cross-cultural study by Buss (1989), 10,047 people from 37 cultures were asked to rate and rank the desirability of several traits in a sexual partner. Among the top 10 most desired traits, by both sexes and across almost all cultures, were kindness, an exciting personality, adaptability, and chastity. Moreover, many studies on lonely hearts personal advertisements in newspapers and magazines showed that people often state that they possess, and are seeking, moral traits, especially kindness, generosity, honesty, fidelity, and the capacity for commitment (e.g., Oda, 2001). There are, however, a number of aspects of morality beyond those representing the holders' quality or ability. For example, as described below, adherence to tradition and submission to legitimate authority, which cannot be considered as initiative of mate quality, are thought to provide the foundation for morality. Although such an aspect of morality may have been maintained by assortative mating, there have been few studies on assortative mating in the context of morality.

In the present study, we investigated whether people feel attracted to potential partners with similar morals to themselves. A variety of views have been presented about what kind of intention, decision, or action is included in the concept of morality. In this study, we adopted the moral foundation theory, which explains the origins of, and variation in, human moral reasoning, based on innate, modular foundations (Graham et al., 2011). Haidt (2012) argued that humans are equipped by the evolutionary process with a set of automatic moral intuitions, but these moral intuitions can be altered by social and cultural influences. The moral foundation theory posits that moral behavior can best be described according to five general moral values: 1) Harm/Care (refers to the virtues of warmheartedness, humaneness, and nurturance, which evolved to elicit a caring and sensitive response required to help those in need), 2) Fairness/Reciprocity (refers to ideas of justice, rights and impartiality, which evolved in response to the adaptive threat of being exploited by cheaters, and increases one's chances of receiving the benefits of cooperation), 3) Ingroup/Loyalty (refers to fidelity, patriotism and self-abnegation in favor of other group 
members, which evolved to maintain group cohesion by making an individual aware of others who may want to hurt or ostracize members of the group), 4) Authority/ Respect (refers to respect for legitimate power, authority and tradition, which evolved in response to maintaining and respecting social hierarchies), and 5) Purity/Sanctity (refers to the abhorrence of disgusting things and contamination, which evolved in response to the danger of pathogens and parasites). Among the morality elements considered in previous studies, cooperation is related to Fairness/Reciprocity and Ingroup/Loyalty, kindness is related to Harm/Care, generosity and honesty are related to Fairness/Reciprocity, and fidelity and chastity are related to Ingroup/Loyalty and Purity/Sanctity. The Moral Foundations questionnaire was developed to measure the five domains of moral foundations (Graham et al., 2011). We evaluated our participants' moral standards with respect to their ideal long-term romantic partners according to these five values, and compared them with their standards for themselves.

In addition to the preference for similarity, another type of preference, "aspirational assortative preference", has been discussed. Aspirational assortative preference suggests that people desire partners with traits that are similar to, but superior to, their own (Watson et al., 2014). People are expected to prefer higher moral standards according to the theory of sexual selection, but this might not apply if the partner's morals are quite different from their own. Accordingly, the morals that people desire in their ideal partners could be anchored by self-appraisals pertaining to moral character. As well as these relative mate preferences, an absolute preference for morality should also be expected. Absolute mate preference is expected to differ between the sexes according to sexual selection theory. In the present study, we compared the magnitude of the preferences for the five moral foundations between the two sexes. However, large sex differences in preferences with regard to the five foundations were not expected to be seen because each foundation is important for both sexes.

In the present study, absolute and relative mate preferences for the Big Five traits were investigated simultaneously to determine whether participants confused the questions about preference for an ideal partner with that about themselves. The former questions were modified from the latter ones and required taking the perspective of an ideal partner. If participants did not fully understand the difference between the two similar questions, a high correlation might cause an artifact. We should obtain, however, a similar pattern in relation to the Big Five preferences to those of previous studies if participants understood the difference between questions on an ideal romantic partner and those relating to themselves. Because of the word limitation, details of the preference study on the Big Five traits were reported in the supplementary.

\section{Methods}

Participants

In total, 185 Japanese undergraduates (101 females, 84 males; mean age: $19.3 \pm 1.3$ years) at two universities participated. They responded to a paper and pencil questionnaire in classrooms and received no monetary reward for their involvement. Informed consent was obtained from all participants included in the study.

Measures

MFQ-SELF: The Japanese version of the 30-item Moral Foundations Questionnaire (MFQ; Kanai, 2019 translated from Graham et al., 2011) was used to assess the moral foundations of the participants. To be consistent with the MFQ-IDEAL, described below, the word "someone" in each item was replaced with the initial of a person, "A". The second section measures the degree to which participants agreed or disagreed with sentences describing moral judgments, using a six-point Likert scale ranging from strongly disagree (0) to strongly agree (5).

MFQ-IDEAL: This version of the MFQ, designed to investigate the moral foundations of one's "ideal romantic partner", is an adapted form of the original MFQ. To create a questionnaire that looked specifically at an individual's ideal romantic partner, in the first section participants were asked to evaluate their ideal romantic partners' morals. As stated above, the word "someone" was replaced with the initial of a person, "A", and the six-point Likert scale was modified to range from not important at all (0) (as a moral trait in an ideal long-term partner) to extremely important (5). That is, we measured not participants' evaluation of the moral relevance of several moral considerations but their evaluation of moral consideration of their ideal romantic partners to a person "A". No other changes were made to the original instrument. To help the participants to understand the changes, a preliminary exercise was completed before they answered the first part of the questionnaire (Figure 1). In the second section, we asked participants to rate the desirability of the moral traits described by the questionnaire items in a romantic partner, using a six-point Likert scale ranging from not desirable at all (0) to strongly desirable (5). No changes were made to the original instrument in this section.

These scales, as well as BFS-SELF and BFS-IDEAL (see the supplementary), were bound into a booklet and administered to participants. It was emphasized in the instructions that the participants should consider their own ideal romantic partner, rather than that which they believed the rest of society might consider ideal. Following Figueredo et al. (2006), the questionnaires on self was administered after the questionnaires on an ideal partner to avoid priming the subjects for intentionally matching the ideal romantic partner's personality to one's own. Moreover, the questionnaire on the Big Five traits was inserted between the MFQ-IDEAL and the MFQ-SELF to prevent confusion. That is, The MFQ-IDEAL was completed first, followed by the BFS-IDEAL, MFQSELF, and then the BFS-SELF. The study was approved by the Bioethics Review Committee of Nagoya Institute of Technology (No. 30-001).

\section{Statistical analyses}

Bivariate correlations between self and ideal romantic partner ratings were calculated for all factors. Self-scores were subtracted from those of the ideal romantic partner factors and tested against zero to identify discrepancies between the ratings of self and ideal romantic partners. A power analysis using $G^{*}$ Power 3.1 showed that a sample of 84 participants was required to compare the correlation with 0 , given an effect size of 0.3 (medium; 


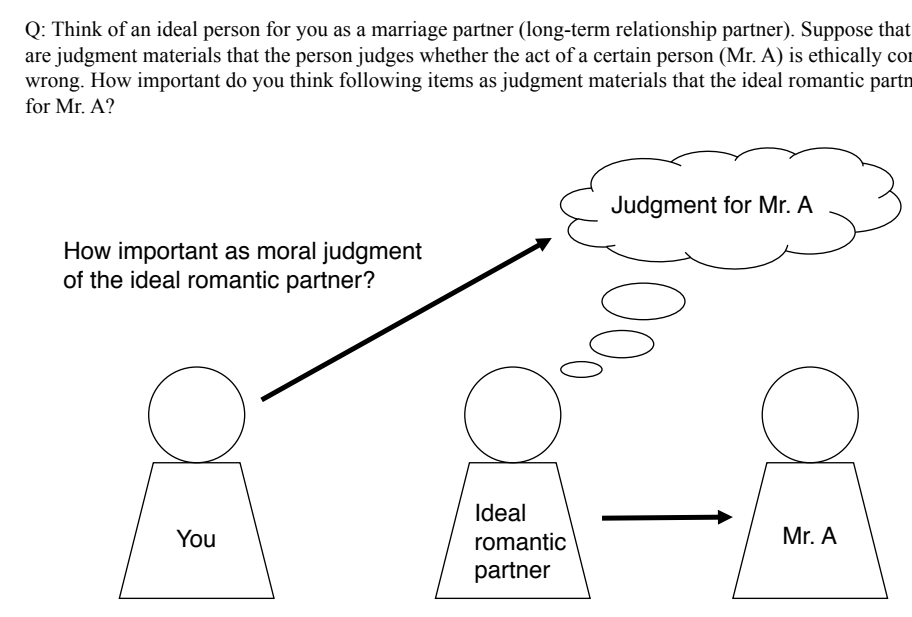

Figure 1. Question and illustration presented for participants to help understanding of the first part of MFQ-IDEAL

Cohen, 1992), power of .80 and an alpha of .05 and that a sample of 64 participants in each group was required to detect a between-group difference, given an effect size of 0.5 (medium; Cohen, 1992), a power of .80 and an alpha of .05 ( $t$-test). Thus, our sample size was sufficient for the analyses performed.

\section{Results}

No significant sex differences were observed in the MFQ-IDEAL or MFQ-SELF scores, with the exception of the Harm score for the MFQ-SELF (Tables 1 and 2). Cronbach's alpha values were lower than those reported by the authors of the scale (Tables 1 and 2). The alpha value for the individualizing moral foundations subscale (including Harm and Fairness) of MFQ-IDEAL was .72 , and that for the binding moral foundations subscale (including Ingroup, Authority, and Purity) was .76. The alpha value for the individualizing moral foundations subscale of MFQ-SELF was .73, and that for the binding moral foundations subscale was 79 .

In both sexes, correlations between self and ideal romantic partner ratings on these same foundations were all rather high and statistically significant (Table 3). Differences between the self-rated scores and those of the ideal romantic partner scores did not deviate significantly from zero (Table 4). There was no sex difference in the magnitude of the differences between self and romantic partner scores (Table 4).

\section{Discussion}

No significant sex differences were found in self-ratings on MFQ traits, except for Harm, or for ratings regarding the long-term ideal romantic partner. Scores on the MFQSELF and MFQ-IDEAL were highly correlated, albeit differences between the MFQ-SELF and MFQ-IDEAL scores did not deviate significantly from zero in any case.

The fact that we found no sex difference in preferred moral traits in the ideal romantic partner suggests that there was no tendency for absolute mate preference in morality. On the other hand, the strong correlation between self-ratings and ratings of the ideal romantic partner pointed to a strong relative mate preference. It is possible that this high correlation might be due to participants not fully understanding the difference between the MFQ-

Table 1. Scores of each factor in MFQ-IDEAL

\begin{tabular}{|c|c|c|c|c|c|c|c|}
\hline & $\begin{array}{c}\text { Cronbach's } \\
\text { alpha }\end{array}$ & $\begin{array}{l}\text { Female } \\
M \pm S D\end{array}$ & $\begin{array}{c}\text { Male } \\
M \pm S D\end{array}$ & $t$ & $d f$ & Cohen's $d$ & $\begin{array}{c}\text { All } \\
M \pm S D\end{array}$ \\
\hline Fairness & .59 & $19.6 \pm 3.6$ & $19.1 \pm 3.7$ & -0.96 & 174.65 & 0.14 & $19.4 \pm 3.6$ \\
\hline Authority & .45 & $15.4 \pm 3.4$ & $14.9 \pm 3.7$ & -0.88 & 171.24 & 0.14 & $15.2 \pm 3.6$ \\
\hline Purity & .42 & $18.4 \pm 3.2$ & $17.9 \pm 3.7$ & -0.97 & 166.95 & 0.15 & $18.1 \pm 3.4$ \\
\hline
\end{tabular}

Note. Degree of freedom was adjusted because Welch $t$-test was employed.

Table 2. Scores of each factor in MFQ-SELF

\begin{tabular}{|c|c|c|c|c|c|c|c|}
\hline & $\begin{array}{c}\text { Cronbach's } \\
\text { alpha }\end{array}$ & $\begin{array}{l}\text { Female } \\
M \pm S D\end{array}$ & $\begin{array}{c}\text { Male } \\
M \pm S D\end{array}$ & $t$ & $d f$ & Cohen's $d$ & $\begin{array}{c}\text { All } \\
M \pm S D\end{array}$ \\
\hline Fairness & .61 & $19.9 \pm 3.8$ & $18.9 \pm 4.1$ & -1.67 & 172.05 & 0.25 & $19.4 \pm 4.0$ \\
\hline Authority & .50 & $15.5 \pm 4.1$ & $15.3 \pm 4.1$ & -0.36 & 175.91 & 0.05 & $15.4 \pm 4.1$ \\
\hline Purity & .54 & $18.5 \pm 3.8$ & $18.0 \pm 3.8$ & -0.95 & 176.67 & 0.13 & $18.3 \pm 3.8$ \\
\hline
\end{tabular}

Note. Degree of freedom was adjusted because Welch $t$-test was employed. $*<.05$ 
Table 3. Correlation between MFQ-SELF and MFQ-IDEAL

\begin{tabular}{lcll}
\hline & Female & Male & All \\
\hline Harm & $.73^{* * *}$ & $.71^{* * * *}$ & $.73^{* * *}$ \\
Fairness & $.67^{* * *}$ & $.80^{* * *}$ & $.73^{* * *}$ \\
Ingroup & $.71^{* * *}$ & $.79^{* * *}$ & $.75^{* * *}$ \\
Authority & $.74^{* * *}$ & $.77^{* * *}$ & $.76^{* * *}$ \\
Purity & $.78^{* * *}$ & $.84^{* * *}$ & $.81^{* * *}$ \\
\hline$* * *<001$ & &
\end{tabular}

$* * *<.001$

Table 4. Mean differences between self and ideal romantic partner on each morality factor

\begin{tabular}{|c|c|c|c|c|c|c|}
\hline & $\begin{array}{l}\text { Female } \\
M \pm S D\end{array}$ & $\begin{array}{c}\text { Male } \\
M \pm S D\end{array}$ & $t$ & $d f$ & Cohen's $d$ & $\begin{array}{c}\text { All } \\
M \pm S D\end{array}$ \\
\hline Harm & $-0.34 \pm 2.45$ & $0.18 \pm 2.93$ & 1.28 & 162.01 & 0.19 & $-0.10 \pm 2.69$ \\
\hline Fairness & $-0.26 \pm 3.02$ & $0.20 \pm 2.49$ & 1.13 & 182.99 & 0.16 & $-0.05 \pm 2.79$ \\
\hline Ingroup & $0.36 \pm 3.18$ & $0.05 \pm 2.55$ & -0.73 & 182.79 & 0.11 & $0.22 \pm 2.91$ \\
\hline Authority & $-0.10 \pm 2.76$ & $-0.35 \pm 2.69$ & -0.61 & 178.32 & 0.09 & $-0.21 \pm 2.72$ \\
\hline Purity & $-0.17 \pm 2.43$ & $-0.13 \pm 2.14$ & 0.11 & 182.30 & 0.02 & $-0.15 \pm 2.30$ \\
\hline
\end{tabular}

Note. Degree of freedom was adjusted because Welch $t$-test was employed.

SELF and MFQ-IDEAL, thus answering questions in the two instruments in the same way. However, correlations between self and ideal romantic partner ratings on the Big Five traits were lower than those for moral foundations, and showed a similar pattern to a previous study (see the supplementary). This suggests that the participants did not confuse the MFQ-SELF with MFQ-IDEAL.

The results suggest that, regardless of differences in the importance of particular moral domains, the level of agreement with one's partner is of primary importance; such agreement could be the basis for maintaining moral sensibility in a group. One problem with these findings was that the Cronbach's alpha values for subscales of the MFQs were lower than those obtained by the authors of the scale, although the values for the inclusive indices were sufficient. Previous studies on non-Western subjects have also reported low alphas in each foundation and a sufficient degree of alphas in the inclusive indices (e.g., Kim et al., 2012; Trups-kalne \& Dimdins, 2017; Yilmaz et al., 2016). These results suggest that the internal consistency of the MFQ depends on the sociocultural context. Murayama and Miura (2019) investigated the validity of the Japanese version of MFQ and reported that, although the five-factor model showed the best fit compared to other models, some problems could be found in the reliability of the five foundational dimensions. In fact, such a low internal consistency is an essential and unavoidable issue of the MFQ because, as Graham et al. (2011) have argued, the scale is composed with priority given to measurements in various situations. Another problem was that we examined only aspirational assortative mating. Further study of married couples is needed on actual assortative mating with respect to morality.

\section{Acknowledgments}

This work was supported by JSPS KAKENHI Grant Number 15K04042.

\section{Supplementary Material}

Electronic supplementary material is available online.

\section{References}

Buss, D. M. (1984). Marital assortment for personality dispositions: assessment with three different data sources. Behavior Genetics, 14, 111-123. https://doi. org/10.1007/BF01076408

Buss, D. M. (1989). Sex differences in human mate selection: evolutionary hypotheses tested in 37 cultures. Behavioral and Brain Sciences, 12, 1-49. https://doi.org/10.1017/S0140525X00023992

Cohen, J. (1992). A power primer. Psychological Bulletin, 112, 155-159. https://doi.org/10.1037/00332909.112.1.155

Figueredo, A. J., Sefcek, J. A., \& Jones, D. N. (2006). The ideal romantic partner personality. Personality and Individual Differences, 41, 431-441. https://doi. org/10.1016/j.paid.2006.02.004

Graham, J., Nosek, B. A., Haidt, J., Iyer, R., Koleva, S., \& Ditto, P. H. (2011). Mapping the moral domain. Journal of Personality and Social Psychology, 101, 366-385. https://doi.org/10.1037/a0021847

Haidt, J. (2012). The righteous mind: why good people are divided by politics and religion. Pantheon Books.

Haidt, J., Koller, S. H., \& Dias, M. G. (1993). Affect, culture, and morality, or is it wrong to eat your dog? Journal of Personality and Social Psychology, 65, 613628. https://doi.org/10.1037/0022-3514.65.4.613

Kanai, R. (2019, November 2). MFQ Translations (Japanese). https://docs.google.com/spreadsheets/ d/1UflzHkc8g5ohW_MIKGzbrGH5bIPiJoWcSvfuq7OsoYc/ edit\#gid=39

Kim, K. R., Kang, J-S., \& Yun, S. (2012). Moral intuitions and political orientation: similarities and differences between Korea and the United States. Psychological Reports, 111, 173-185. https://doi.org/10.2466/17.09.21. PR0.111.4.173-185

Laland, K. N., Odling-Smee, J., \& Feldman, M. W. (2001). Cultural niche construction and human evolution. Journal of Evolutionary Biology, 14, 22-33. https://doi. org/10.1046/j.1420-9101.2001.00262.x

McElreath, R., Boyd, R., \& Richerson, P. J. (2003). Shared norms and the evolution of ethnic markers. Current Anthropology, 44, 122-130. https://doi. org $/ 10.1086 / 345689$

McPherson, M., Smith-Lovin, L., \& Cook, J. M. (2001). Birds of a feather: homophily in social networks. Annual Review of Sociology, 27, 415-444. https://doi. org/10.1146/annurev.soc.27.1.415

Miller, G. F. (2007). Sexual selection for moral virtues. The Quarterly Review of Biology, 82, 97-125. https:// doi.org/10.1086/517857

Murayama, A., \& Miura, A. (2019). Validation of the 
Japanese version of Moral Foundation Questionnaire: investigating relationship with ideologies. Shinrigakukenkyu [The Japanese Journal of Psychology], 90, 156-166 [in Japanese with an English abstract]. https://doi.org/10.4992/jjpsy.90.17234

Oda, R. (2001). Sexually dimorphic mate preference in Japan: an analysis of lonely hearts advertisements. Human Nature, 12, 191-206. https://doi.org/10.1007/ s12110-001-1006-x

Trups-kalne, I., \& Dimdins, G. (2017). Relation between social conservatism, moral competence, moral orientations, and the importance of moral foundations. Mediterranean Journal of Social Sciences, 8, 81-90. https://doi.org/10.1515/mjss-2017-0044

Watson, D., Beer, A., \& McDade-Montez, E. (2014). The role of active assortment in spousal similarity. Journal of Personality, 82, 116-129. https://doi.org/10.1111/ jopy. 12039

Yamagishi, T., \& Hashimoto, H. (2016). Social niche construction. Current Opinion in Psychology, 8, 119124. https://doi.org/10.1016/j.copsyc.2015.10.003

Yilmaz, O., Harma, M., Bahçekapili, H. G., \& Cesur, S. (2016). Validation of the Moral Foundations Questionnaire in Turkey and its relation to cultural schemas of individualism and collectivism. Personality and Individual Differences, 99, 149-154. https://doi.org/10.1016/j.paid.2016.04.090 